\title{
NONLINEAR CONTROL DESIGN AND AVERAGING ANALYSIS OF FULL-BRIDGE BOOST RECTIFIER
}

\author{
A. Abouloifa, F. Giri*, I. Lachkar, F.Z. Chaoui**, and Robert Griñó*** \\ *GREYC, Université De Caen, France,E-mail: abouloifa@yahoo.fr, giri@greyc.ensicaen.fr \\ ** LA2I, EMI, Rabat, Morocco,E-mail: ibtissamlachk74@yahoo.fr, chaouifatima@yahoo.fr \\ *** IOC, UPC, Barcelona, Spain, E-mail: grino@ioc.upc.es
}

Abstract: We are considering the problem of controlling AC/DC full bridge converters. The control objectives are twofold: (i) guaranteeing a regulated voltage for the supplied load, (ii) enforcing power factor correction (PFC) with respect to the main supply network. The considered problem is dealt with using a nonlinear controller that involves two loops in cascade. The inner-loop is designed, using sliding mode approach, to cope with the PFC issue. The outer-loop is designed to regulate the converter output voltage. While several double-loop regulators (designed for different converters) can be found in the relevant literature, it is the first time that a so formal average analysis is developed that rigorously describes the controller performances. The development of such theoretical analysis framework is a major motivation of this paper.

\section{INTRODUCTION}

In order to meet the requirements of the electrical quality standards (for example IEC 1000-3-2) concerning the input current entering low-power equipments, it is necessary to perform the AC-DC electrical power conversion using switch-mode power converters [9]. Among these circuits, the most popular for average and high power applications is the boost power converter operating in continuous conduction mode $[1,14]$. From a control viewpoint, such AC/DC converter is viewed as a nonlinear, nonminimum phase and hybrid system. Then, undesirable current harmonics may be generated when the converter is connected to an $\mathrm{AC}$ power source. These harmonics may be harmful for both the converter and the main supply network necessitating additional protection and over-dimensioning of both the converter components and the network elements (transformers, condensers ...). These precautions are costly (higher component prices, higher power consumption).

To avoid the above drawback, the converter should be controlled bearing in mind not only output voltage regulation but also rejection of undesirable current harmonics. The last objective is referred to power factor correction (PFC). A comprehensive overview on circuits that are able (if well controlled) to achieve the PFC requirement can be found in, e.g. [10], [2], [3]. The question of how to achieve simultaneously the PFC requirement and the output regulation objective has been discussed in many early works. The proposed solutions involved the same control strategy:

(i) a current loop is used to achieve the PFC by acting on the switch position;

(ii) a voltage loop is resorted to achieve output voltage regulation through the tuning of the reference of the current loop.

However, no formal analysis was developed in the above works to show that the proposed double-loop regulators actually achieve the performances they were designed for. As a matter of fact, this is not surprising as the proposed regulators are linear (typically PID regulators) while the controlled power converters are highly nonlinear and time-varying. It is only recently that a serious attempt has been made to build up a formal framework for the above double-loop control strategy [6]. There, the backstepping control technique was used to design the involved regulation loops. However, the developed analysis was not complete and not fully rigorous e.g. the PFC requirement has not really been guaranteed (this is detailed later in theorem 1).

In the present paper, we aim at designing a doubleloop regulator for which we can develop a complete and rigorous analysis of the closed-loop performances. To this end, the sliding-mode control technique will be resorted 
to design a double-loop control system. The resulting closed-loop system turns out to be a time-varying and highly nonlinear. In particular, the time-varying feature results in output voltage ripples and the question is: how small the ripples amplitude is? It is worth noting that the averaging theory is the natural framework to analysing such an issue, due to the periodic nature of the closed-loop system. Making a judicious use of averaging results (e.g. $[7,8])$, it will be established that the PFC requirement is effectively achieved and the output ripples are actually insignificant. More precisely, the output tracking error will be shown to be, in steadystate, a harmonic signal whose amplitude depends on the frequency supply-net voltage: the larger is the net frequency, the smaller the tracking error. It is the first time that the insignificance of the output ripples is so formally described.

The paper is organized as follows: Section 2 presents the model of the bidirectional boost rectifier and the control objectives. Section 3 is devoted to designing and analyzing the regulator. The closed-loop performances are illustrated in Section 4. A conclusion and reference list end the paper.

\section{CONTROL PROBLEM FORMULATION}

\subsection{Physical Model of the Boost Converter}

The boost converter (Fig. 1) is described by the following average model [4]:

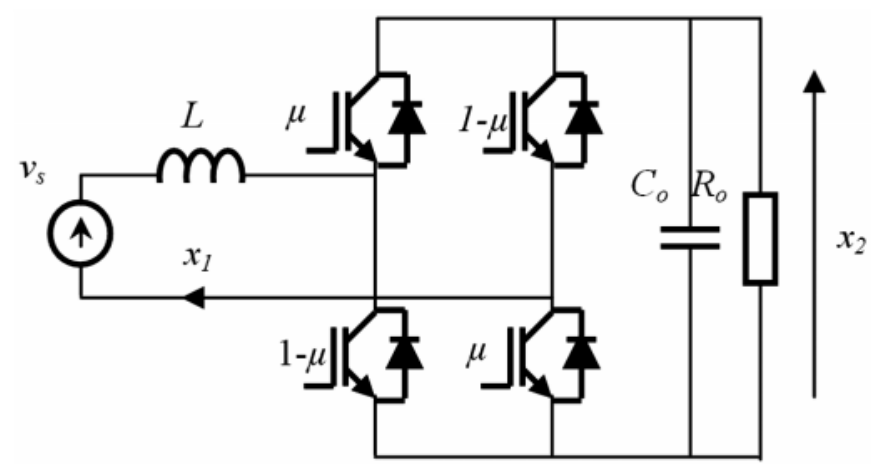

Fig. 1: Bidirectional Boost Active Rectifier Converter

$$
\dot{x}=f(x)+g(x) \mu
$$

with:

$$
f(x)=\left(\begin{array}{c}
\frac{x_{2}+v_{s}}{L} \\
-\frac{x_{1}}{C_{o}}-\frac{x_{2}}{R_{o} C_{o}}
\end{array}\right), \quad g(x)=\left(\begin{array}{c}
\frac{-2 x_{2}}{L} \\
\frac{2 x_{1}}{C_{o}}
\end{array}\right)
$$

where $x_{1}$ and $x_{2}$ denote the average values, over cutting periods, of the input inductor current and the output capacitor voltage variables, respectively; $v_{s}=\hat{V} \sin \left(\omega_{s} t\right)$ is the ideal sinusoidal source that represents the AC-line source; $R_{o}$ is the DC-side resistive load and $L$ and $C_{o}$ are the converter inductance and capacity. The control variable $\mu$ takes its value in the closed real interval $[0,1]$ and represents the average value of the PWM (pulsewidth-modulated) control signal $u \in\{0,1\}$ injected into the real system.

\subsection{Control Objectives}

The control objectives are:

1. The AC-DC converter must operate with a power factor close to one. This amounts to ensuring that, in steady-state, the inductor current $x_{1}$ follows a sinusoidal signal $x_{1}^{d}$ with the same frequency and phase as the AC-line voltage source $v_{s}$, i.e. $x_{1}^{d}=\beta v_{s}(t)$ where $\beta$ is a time real function that should converge to a constant.

2. The DC component of the output capacitor voltage $x_{2}$ should be driven to the constant reference value $x_{2}^{d}$, where $x_{2}^{d}>\hat{V}$ in order to meet the boost feature.

\section{CONTROLLER DESIGN}

The controller synthesis is carried out in two major steps. First, a current inner loop is designed to cope with the PFC issue. In the second step, an outer loop is built-up to achieve voltage regulation.

\subsection{Current Loop Design}

The PFC objective means that the current entering the converter should be sinusoidal and in phase with the AC supply voltage. We therefore seek a regulator that enforces the current $x_{1}$ to track a reference signal $x_{1}^{d}$ of the form:

$$
x_{1}^{d}=\beta v_{s}
$$

At this point the parameter $\beta$ is any function of time that converges to a constant and positive limit. The (inner) control loop will now be designed using the sliding mode approach (e.g. [7]). The first step is the choice of a sliding mode surface. Bearing in mind the control objective (for the inner loop), the choice $h(x, t)=0$ is convenient, with:

$$
h(x, t)=x_{1}-\beta \hat{V} \sin \left(\omega_{s} t\right)
$$

The second step in the sliding-mode approach consists in determining the so-called equivalent control $\mu_{e q}$. This is the control signal that keeps the state vector $x$ in the sliding-surface (whenever the state gets to that surface). Therefore, $\mu_{e q}$ is obtained solving the following equation $\dot{h}(x, t)=0$ with: 


$$
\dot{h}(x, t)=\frac{\partial h(x, t)}{\partial x}(f(x)+g(x) \mu(x))+\frac{\partial h(x, t)}{\partial t} \ldots
$$

Then, $\dot{h}(x, t)=0$ can be rewritten as:

$$
L_{f} h(x, t)+L_{g} h(x, t) \mu+\frac{\partial h(x, t)}{\partial t}=0
$$

Solving this with respect to $\mu$ yields the equivalent control:

$$
\mu_{e q}=-\frac{L_{f} h(x, t)+\frac{\partial h(x, t)}{\partial t}}{L_{g} h(x, t)}
$$

From (1b) it follows that:

$$
\begin{aligned}
L_{f} h(x, t) & =\frac{x_{2}+v_{s}(t)}{L} \\
L_{g} h(x, t) & =\frac{-2 x_{2}}{L} \\
\frac{\partial h(x, t)}{\partial t} & =-\beta \hat{V}_{s} \omega_{s} \cos \left(\omega_{s} t\right)-\dot{\beta} \hat{V}_{s} \sin \left(\omega_{s} t\right)
\end{aligned}
$$

Substituting (6b) in (6a), one gets the following explicit version of the equivalent control signal:

$$
\mu_{e q}=\frac{1}{2}+\frac{\hat{V}}{2 x_{2}}\left[(1-L \dot{\beta}) \sin \left(\omega_{s} t\right)-\beta L \omega_{s} \cos \left(\omega_{s} t\right)\right]
$$

The third step in the sliding-mode technique consists in determining a transient control action, denoted $\mu_{T}$, that is necessary to add to the continuous component $\mu_{e q}$ (equivalent control), so that the obtained total action, namely:

$$
\mu=\mu_{e q}+\mu_{T}
$$

is able to steer the state from any initial position to the sliding surface. To obtain $\mu_{T}$, let us consider the following Lyapunov function

$$
\vartheta(x, t)=0.5 h^{2}(x, t)
$$

Time-derivation of $\vartheta$ gives $\dot{\vartheta}=h(x, t) L_{g} h(x, t) \mu_{T}$, where we have used (1a), (3), (7) and (8). This shows that it would be convenient letting $\mu_{T}$ be of the form:

$$
\mu_{T}=-\tilde{\mu} / L_{g} h(x, t)_{T}
$$

Doing so, one gets:

$$
\dot{\vartheta}=-h(x, t) \tilde{\mu}_{T}
$$

This suggests the following choice of $\tilde{\mu}_{T}$ :

$$
\tilde{\mu}_{T}=k \hat{V} S(h(x, t))
$$

where $k$ is any positive constant real and $S(\cdot)$ is any real function such that:

$$
\xi S(\xi)>0(\text { for all } \xi \neq 0 \text { ) and } S(0)=0
$$

Actually, the substitution of (12) to $\widetilde{\mu}_{T}$ in (11) yields:

$$
\dot{\vartheta}=-k \hat{V} h(x, t) S(h(x, t))
$$

which clearly implies that $\dot{\vartheta}$ is a negative-definite function of $h$. As $\vartheta$ is a positive-definite function of $h$, it follows that the surface $h(x, t)=0$ is globally attractive. In the latter, the following choice will be made for the function $S(\cdot)$ :

$$
S(h(x, t))=(2 / \pi) \arctan (h(x, t) / \eta)
$$

where $\eta$ is any small positive constant real. But, note that any other choice can also be made provided that the above mentioned conditions are fulfilled.

Now, substituting (7), (10) and (12) in (8) gives the following control law:

$$
\mu=\frac{1}{2}+\frac{\hat{V}}{2 x_{2}}\left(\begin{array}{l}
(1-L \dot{\beta}) \sin \left(\omega_{s} t\right)-\beta L \omega_{s} \\
\cos \left(\omega_{s} t\right)+L k S(h(x, t))
\end{array}\right)
$$

Finally, substituting (13) to $\mu$ in (4) gives:

$$
\dot{h}=-k \hat{V} S(h)
$$

The results thus established is summarized in the following proposition.

Proposition 1: Consider the control system, next called inner closed-loop, consisting of the system (1a-b) and the control law (13). If the ratio $\beta$ and its first derivatives are available then:

1. The inner closed-loop system undergoes, in the $h(t)$-coordinate, Eq. (14) and this is globally asymptotically stable with respect to the Lyapunov function $\vartheta(x, t)=0.5 h^{2}(x, t)$. Consequently, the function $h(x, t)$ vanishes asymptotically, whatever the initial conditions are.

2. If in addition $\beta$ is constant, or is varying but converges to a positive limit value, then the PFC requirement is fulfilled.

\subsection{Voltage Loop Design}

The aim of the outer loop is to generate the function $\beta$ in such a way that the output voltage $x_{2}$ be regulated to a given reference value $x_{2}^{d}$. To first step is to establish the 
relation between $\beta$ (the outer loop control signal) and the squared-voltage $y=x_{2}^{2}$.

\subsubsection{Relation between $b$ and $y=x_{2}^{2}$}

This is established in the following proposition.

Proposition 2: Consider the power converter described by $(1 a-b)$ in closed-loop with the inner control law defined by (13). Under the same assumptions as in Proposition 1, one has following relation between $y=x_{2}^{2}$ and $\beta$ :

$$
\begin{aligned}
\frac{d y(t)}{d t}+a y(t) & \\
= & k_{o} \beta(1-L \dot{\beta})-k_{o} \beta f(\beta) \cos \left(2 \omega_{s} t-\varphi(\beta)\right)+ \\
& \frac{2 L k \hat{V}}{C_{o}}\left(h+\beta \hat{V} \sin \left(\omega_{s} t\right)\right) S(h(x, t))+ \\
& \frac{2 \hat{V} h}{C_{o}} f(\beta) \sin \left(\omega_{s} t-\varphi(\beta)\right)
\end{aligned}
$$

with $\frac{d h}{d t}=-k \hat{V} S(h)($ due to $(14))$ and:

$$
\begin{aligned}
& f(\beta)=\sqrt{(1-L \dot{\beta})^{2}+\left(\beta L \omega_{s}\right)^{2}} \\
& \varphi(\beta)=\arctan \left(\frac{\beta L \omega_{s}}{1-L \dot{\beta}}\right), k_{o}=\frac{\hat{V}^{2}}{C_{o}}
\end{aligned}
$$

Proof: Multiplying both sides of the second equation in (1a) by $2 x_{2}$ yields:

$$
\begin{aligned}
& \frac{d y(t)}{d t}+a y(t) \\
&=2 \frac{2 \mu-1}{C_{o}} x_{2}(t) x_{1}(t)
\end{aligned}
$$

On the other hand, one gets from (3) $x_{1}=h(x, t)+$ $\beta \hat{V} \sin \left(\omega_{s} t\right)$. Now, replacing in Eq. (16) $x_{1}$ by $h(x, t)+\beta \hat{V}$ $\sin \left(\omega_{s} t\right)$ and $\mu$ by (13) one gets (15).

\subsubsection{Squared Output Voltage Regulation}

The function $\beta$ stands for the control signal in the system described by (14)-(15). The problem at hand is to generate $\beta$ so that the squared voltage $y=x_{2}^{2}$ tracks the reference signal $y^{d} \hat{=}\left(x_{2}^{d}\right)^{2}$. Bearing in mind the fact that $\beta$ and its first derivatives should be available (Proposition 1), a filtered PI type control law is proposed, namely:

$$
\beta=\left(\frac{b}{b+s}\right)\left(k_{p} e_{1}+k_{i} e_{2}\right)
$$

$$
\begin{aligned}
& e_{1}=y^{d}-y, \quad e_{2}=\int_{0}^{t} e_{1} d t, y=x_{2}^{2}, \\
& y^{d}=\left(x_{2}^{d}\right)^{2}
\end{aligned}
$$

where $s$ may denote as well the Laplace variable or the derivative operator $(s=d / d t)$, depending on the context. At this point, the regulator parameters $\left(b, k_{p}, k_{i}\right)$ are any positive real constants. The next analysis will make it clear how these should be chosen for the control objectives to be achieved.

\subsection{Control System Analysis}

In the following Theorem, it is shown that, for a class of reference signals, the control objectives are achieved (in the mean) with an accuracy that depends on the network frequency $\omega_{s}$. The following notations are needed to formulate the results:

$$
\begin{aligned}
\varepsilon= & 1 / \omega_{s}, \quad a_{o}=\frac{2 k k_{o} k_{i} b \hat{V}}{\pi \eta} \\
a_{1}= & \frac{2 k \hat{V}}{\pi \eta}\left(a\left(1-L b k_{p} y^{r}\right)+b\left(a+k_{p} k_{o}\right)\right)+b k_{o} k_{i} \\
a_{2}= & \frac{2 k \hat{V}}{\pi \eta}\left(b+a\left(1-L b k_{p} y^{r}\right)\right)+ \\
& a b\left(1+L k_{i} y^{r}\right)+b k_{p} k_{o} \\
a_{3}= & \frac{2 k \hat{V}}{\pi \eta}+b+a\left(1-L b k_{p} y^{r}\right)
\end{aligned}
$$

Theorem 1 (main result). Consider the AC/DC Boost power converter shown by Fig.1, represented by its average model $(1 a-b)$, together with the controller consisting of the inner-loop regulator (13) and the outerloop regulator (17). Then, the closed-loop system has the following properties:

1. The error $h=x_{1}-x_{1}^{d}$ vanishes asymptotically, where $x_{1}^{d}=\beta v_{s}$.

2. Let the reference signal $x_{2}^{d}>\hat{V}$ be periodic with period $N \pi / \omega_{s}$, where $N$ is any positive integer. Let the regulator parameters $\left(b, k_{p}, k_{i}\right)$ be any positive real numbers that satisfy the following inequalities:

$$
\begin{aligned}
& a_{3}>0 \\
& a_{2} a_{3}-a_{1}-a_{3}>0 \\
& a_{1} a_{2} a_{3}-a_{1}^{2}-a_{0} a_{3}^{2}+a_{1}-a_{2} a_{3}>0
\end{aligned}
$$

Then, there exists a positive real $\varepsilon^{*}$ such that if $\varepsilon \leq \varepsilon^{*}$, one has: 
(a) The tracking error $e_{1}$ and $\beta$ are harmonic signals that continuously depend on $\varepsilon$, i.e.

$$
e_{1}=e_{1}(t, \varepsilon) \text { and } \beta_{1}=\beta_{1}(t, \varepsilon)
$$

(b) Furthermore, the above signals satisfy:

$$
\text { (i) } \lim _{\varepsilon \rightarrow 0} e_{1}(t, \varepsilon)=0 ; \quad \text { (ii) } \lim _{\varepsilon \rightarrow 0} \beta(t, \varepsilon)=\frac{a \bar{y}^{d}}{k_{o}}
$$

where $\bar{y}^{d}$ denotes the mean value of the periodic reference signal $y^{d}$.

Proof: Proof of Part 1: Equation (17) guarantees that $\beta$ and its derivatives are available. Then, Part 1 of the Theorem follows directly from Proposition 1 . This also guarantees that Eq. (15), in Proposition 2, holds.

Proof of Part 2: In order to prove the second part of Theorem 1, let us introduce the following state variables:

$$
\begin{gathered}
x_{0,1}=e_{1}, x_{0,2}=e_{2}, x_{0,3}=\frac{b}{b+s}\left(k_{p} e_{1}+k_{i} e_{2}\right), \\
x_{0,4}=h
\end{gathered}
$$

It follows from (15), (17) and (20) that the state vector undergoes the following state equation:

$$
\frac{d X_{o}}{d t}=f\left(t, X_{o}, y^{d}\right)
$$

where:

$$
f\left(t, X_{o}, y^{d}\right)=\left(\begin{array}{c}
\psi \\
x_{0,1} \\
b\left(k_{p} x_{0,1}+k_{i} x_{0,2}-x_{0,3}\right) \\
-k \hat{V} \operatorname{sgn} d\left(x_{0,4}\right)
\end{array}\right)
$$

with:

$$
\begin{aligned}
\psi= & a y^{d}+\frac{d y^{d}}{d t}-a x_{0,1}-k_{o} x_{0,3}+ \\
& L b k_{o} k_{p} x_{0,1} x_{0,3}+L b k_{o} k_{i} x_{0,2} x_{0,3}- \\
& L b k_{o} x_{0,3}^{2}+k_{o} x_{0,3} f\left(x_{0,3}\right) \\
& \cos \left(2 \omega_{s} t-\varphi\left(x_{0,3}\right)\right)-\frac{2 \hat{V} x_{4}}{C_{o}} f\left(x_{0,3}\right) \\
& \sin \left(\omega_{s} t-\varphi\left(x_{0,3}\right)\right)-\frac{2 L k \hat{V}}{C_{o}} \\
& \left(x_{0,4}+x_{0,3} \hat{V} \sin \left(\omega_{s} t\right)\right) S
\end{aligned}
$$

Stability of the above system will now be dealt with using averaging theory. As $y^{d}$ is periodic with period
$N \pi / \omega_{s}$ it will prove to be useful introducing the following auxiliary reference function:

$$
y^{r}(t)=y^{d}\left(N t / \omega_{s}\right)
$$

This readily implies that $y^{d}(t)=y^{r}\left(\omega_{s} t / N\right)$ and that $y^{r}$ is periodic, with period $2 \pi$. Let us now introduce the time-scale change $\tau=\omega_{s} t$. Then, the term containing $y^{d}$ in $\psi$ becomes:

$$
\begin{aligned}
& a y^{d}(t)+\frac{d y^{d}(t)}{d t} \\
& \quad=a y^{r}(\tau / N)+\omega_{s} \frac{d y^{r}(\tau / N)}{d \tau}
\end{aligned}
$$

It also follows from (21)-(22) that $Z_{o}(\tau) \hat{\equiv} X_{o}\left(\tau / 2 \omega_{s}\right)$ undergoes the differential equation:

$$
\frac{d Z_{o}}{d \tau}=\varepsilon g\left(\tau, Z_{o}, \varepsilon\right)
$$

where $(i=1,2,3,4)$ denote the components of the vector $Z_{o}$ and:

$$
g\left(\tau, Z_{o}, \varepsilon\right)=\left(\begin{array}{c}
\psi_{\tau} \\
z_{0,1} \\
b\left(k_{p} z_{0,1}+k_{i} z_{0,2}-z_{0,3}\right) \\
-k \hat{V} \operatorname{sgn} d\left(z_{0,4}\right)
\end{array}\right)
$$

with

$$
\begin{aligned}
\psi_{\tau}= & a y^{r}+\omega_{s} \frac{d y^{r}}{d \tau}-a z_{0,1}-k_{o} z_{0,3}+ \\
& L b k_{o} k_{p} z_{0,1} z_{0,3}+L b k_{o} k_{i} z_{0,2} z_{0,3}-L b k_{o} z_{0,3}^{2}+ \\
& k_{o} z_{0,3} f\left(z_{0,3}\right) \cos \left(2 \tau-\varphi\left(z_{0,3}\right)\right)- \\
& \frac{2 \hat{V} x_{4}}{C_{o}} f\left(z_{0,3}\right) \sin \left(\tau-\varphi\left(z_{0,3}\right)\right)- \\
& \frac{2 L k \hat{V}}{C_{o}}\left(z_{0,4}+z_{0,3} \hat{V} \sin (\tau)\right) S\left(z_{0,4}\right) \ldots(26 \mathrm{~b})
\end{aligned}
$$

Now, let us introduce the average function $G(\cdot)$ defined by:

$$
G(W)=\lim _{\varepsilon \rightarrow 0} \frac{1}{2 \pi \mathrm{N}} \int_{0}^{2 \pi N} g(\tau, W, \varepsilon) d \tau
$$

It follows from (26) that: 


$$
G(W)=\left(\begin{array}{c}
\bar{\psi}_{\tau} \\
w_{0,1} \\
b\left(k_{p} w_{0,1}+k_{i} w_{0,2}-w_{0,3}\right) \\
-k \hat{V} S\left(w_{0,4}\right)
\end{array}\right)
$$

with

$$
\begin{aligned}
\bar{\Psi}_{\tau}= & a \bar{y}^{r}-a w_{0,1}-k_{o} w_{0,3}+L b k_{o} k_{p} w_{0,1} w_{0,3}+ \\
& L b k_{o} k_{i} w_{0,2} w_{0,3}-L b k_{o} w_{0,3}^{2}- \\
& \frac{2 L k \hat{V}}{C_{o}} w_{0,4} S\left(w_{0,4}\right)
\end{aligned}
$$

where $(i=1,2,3,4)$ denote the components of the vector $W$ and $\bar{y}^{r}$ denotes the mean value of $y^{r}$ (which is the same as that of $\left.y^{d}\right)$. Note that the mean value over $[0,2 N \pi]$, of the derivative in the first line of $(26 \mathrm{~b})$ is zero because $y^{r}$ is periodic with period $2 \pi$.

In order to get stability results regarding the system of interest (25)-(26), it is sufficient (thanks to averaging theory) to analyze the following averaged system:

$$
\frac{d W}{d t}=\varepsilon G(W)
$$
brium at:

To this end, notice that (29) has a unique equili-

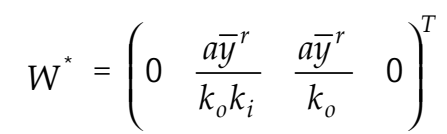

The stability properties of this equilibrium can be checked through the analysis of the linearized version of (29). The stability of the latter is fully determined by its state-matrix:

$$
\begin{aligned}
A & =\left.\frac{\partial G\left(Z_{o}\right)}{\partial Z_{O}}\right|_{W=W^{*}} \\
& =\left(\begin{array}{cccc}
-a\left(1-L b k_{p} \bar{y}^{r}\right) & a b L k_{i} \bar{y}^{r} & -\left(k_{o}+a b L \bar{y}^{r}\right) & 0 \\
1 & 0 & 0 & 0 \\
b k_{p} & b k_{i} & -b & 0 \\
0 & 0 & 0 & -\frac{2 k \hat{V}}{\eta \pi}
\end{array}\right)
\end{aligned}
$$

More specifically, the equilibrium $W^{*}$ is stable provided that the matrix $A$ is Hurwitz. It readily follows from (31) that the eigenvalues of the matrix $A$ are the zeros of the following polynomial:

$\operatorname{det}\left(\lambda I-A_{1}\right)=\lambda^{4}+a_{3} \lambda^{3}+a_{2} \lambda^{2}+a_{1} \lambda^{1}+a_{o}$

where the $a_{i}$ 's are defined by (18). Applying for instance the well known Routh's algebraic criteria, it follows that all zeros of the polynomial (32) have negative real parts if the coefficients $\left(a_{0}-a_{3}\right)$ satisfy (19). Now, invoking averaging theory (e.g. Theorem 4.1 in [8]), one concludes that there exists a $\varepsilon^{*}>0$ such that for $\varepsilon<\varepsilon^{*}$, the differential Eqs (25)-(26) has a harmonic solution $X_{o}=X_{o}(t, \varepsilon)$, that continuously depends on $\varepsilon$, and that:

$$
\lim _{\varepsilon \rightarrow 0} X_{o}(t, \varepsilon)=W^{*}
$$

Then, it readily follows fact that $e_{1}(t, \varepsilon)=x_{0,1}(t, \varepsilon)$ and $e_{2}(t, \varepsilon)=x_{0,2}(t, \varepsilon)$ are in turn harmonic and depend continuously on $\varepsilon$. Then it follows from (17) that $\beta$ is in turn harmonic and depends continuously on $\varepsilon$. Theorem 1 is thus established.

\section{NUMERICAL SIMULATIONS}

\subsection{Experimental Setup}

The performances of the proposed controller are now numerically evaluated using a PWM rectifier with the following characteristics:

Table 1

System Parameters

\begin{tabular}{lcc}
\hline \multicolumn{1}{c}{ Parameters } & & Values \\
\hline Network & $v_{s}$ & $220 \mathrm{~V}, 50 \mathrm{~Hz}$ \\
\hline Converter & $L$ & $1 \mathrm{mH}$ \\
& $r$ & $40 \mathrm{~m} \Omega$ \\
& $C_{o}$ & $4.7 \mathrm{mF}$ \\
& $R_{o}$ & $100 \Omega$ \\
\hline Current & $K$ & 100 \\
Regulator & $\eta$ & 0.1 \\
\hline Voltage & $k_{p}$ & $1.510^{-6}$ \\
Regulator & $k_{i}$ & $1.510^{-5}$ \\
& $b$ & 1000 \\
\hline
\end{tabular}

\subsection{Experimental Protocol}

The simulations aim at illustrating the behavior of the controller in response to step changes on both the voltage reference $x_{2}^{d}$ and the load resistance $R_{0}$. More specifically, the voltage reference goes from $400 \mathrm{~V}$ to $500 \mathrm{~V}$. The load resistance steps from its nominal value $(100 \Omega)$ 
up to infinity (load less) and then down to its nominal value.

\subsection{Simulation Result}

The controller performances are illustrated by Figs 2 to 7. As expected by Theorem 1, the output voltage $x_{2}$ converges in the mean to its reference value with a good accuracy (Fig. 2). Furthermore, it is observed that the voltage ripples oscillates at the frequency $2 \omega_{s^{\prime}}$ but their amplitude is too small compared to the average value of the signals, confirming thus Theorem 1 (Part 2-b-i). The corresponding input current $x_{1}$ is shown in Fig. 3. Finally, Fig. 4 shows that the input current $x_{1}$ and the network voltage $v_{s}$ are actually in phase. Hence, the converter

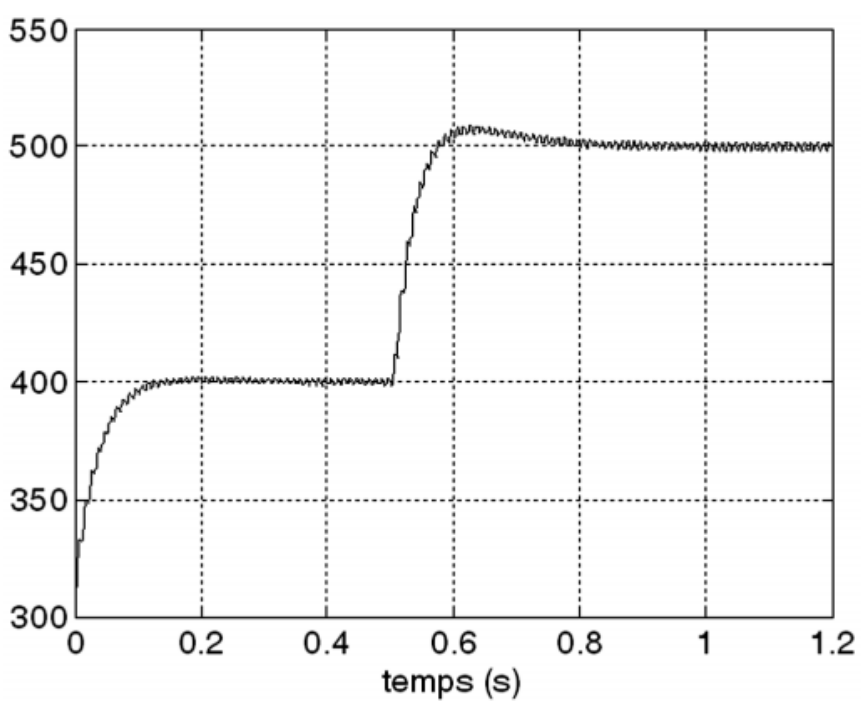

Fig. 2: Output Voltage

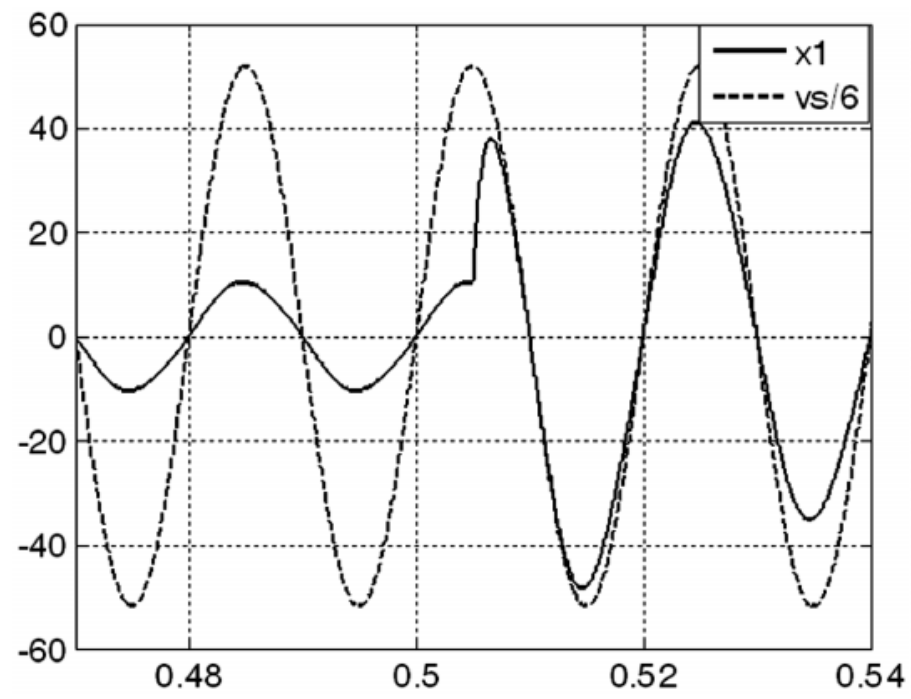

Fig. 4: Unity Power Factor Checking connection to the supply network is done with a unitary power factor. This is further demonstrated by Fig. 5 which shows that the ratio $\beta$ always takes a constant value, after the transient periods that results from the reference voltage steps

Figures 6 to 7 illustrate the behavior of the control system in presence to load changes that are not accounted for in the controller design. The rest of the converter characteristics are kept unchanged. It is seen from Fig. 6 that the disturbing effect due to load changes is well compensated by the controller. Furthermore, Fig. 7 shows the correlation of the current amplitude with the output voltage. Robustness of the proposed controller with respect to load changes is thus established.

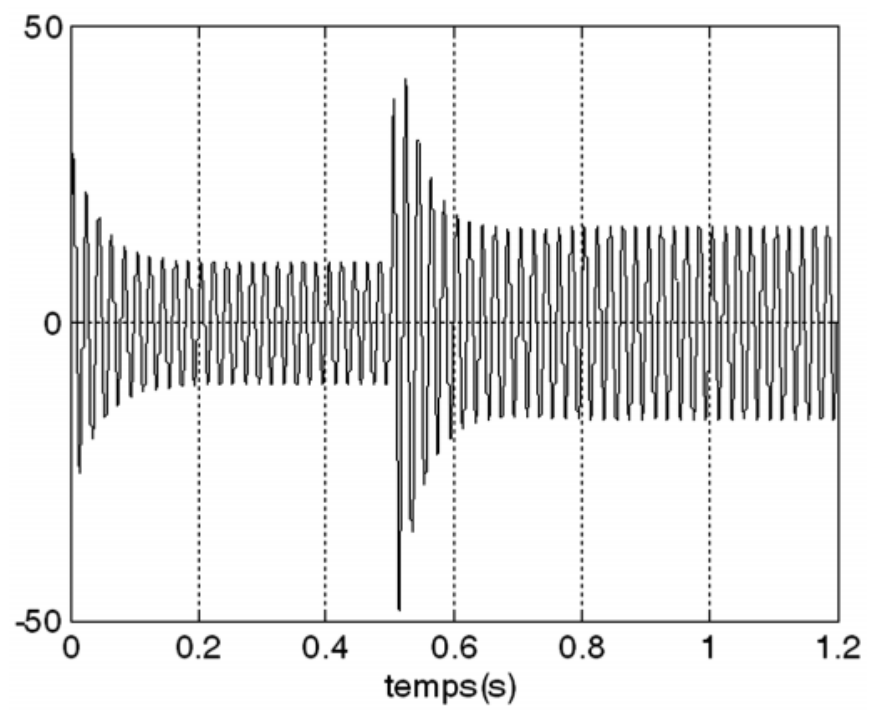

Fig. 3: Input Current $x_{1}$

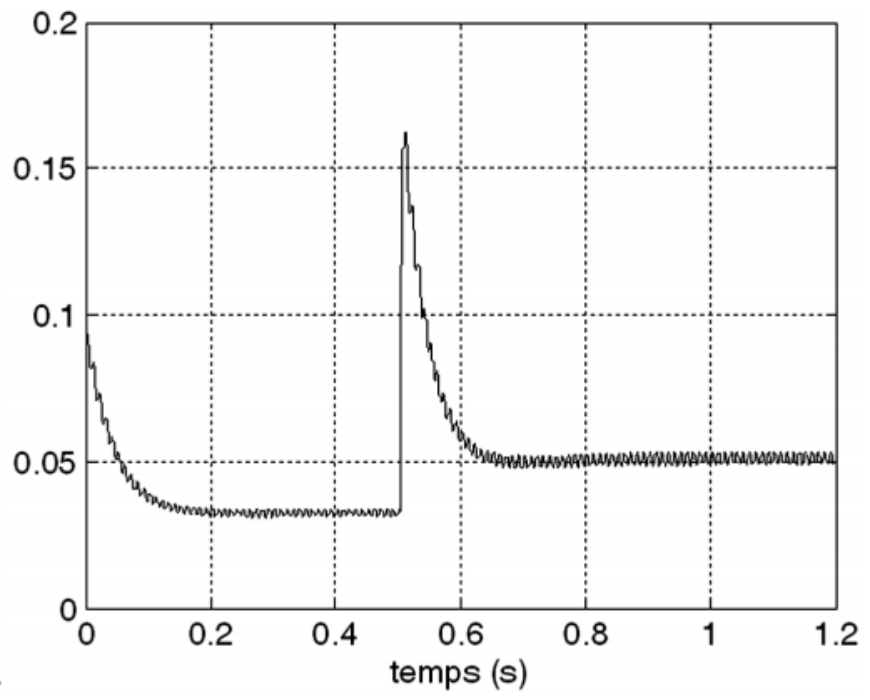

Fig. 5: Signal $\beta$ 


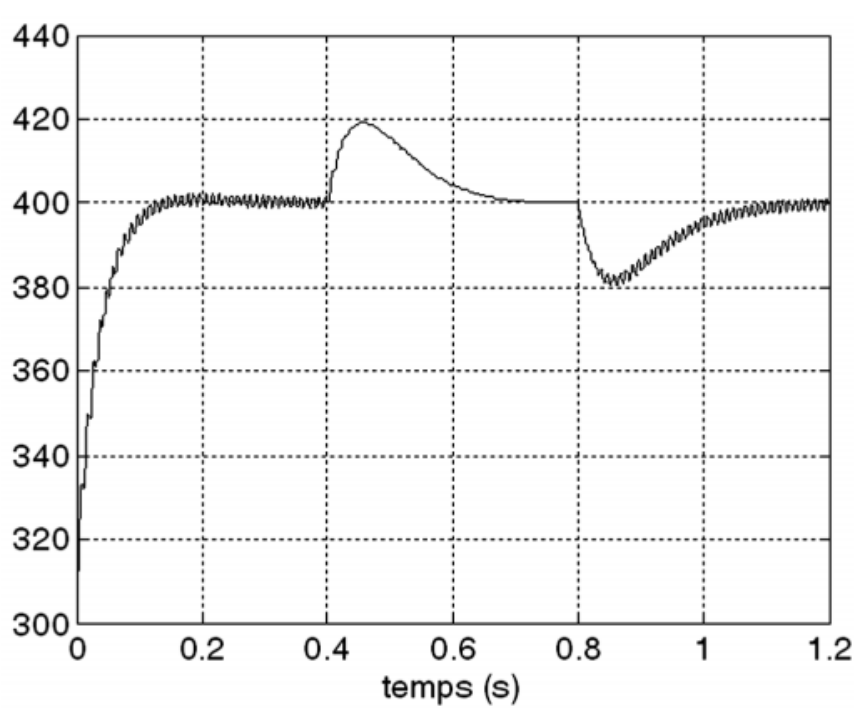

Fig. 6: Output Voltage

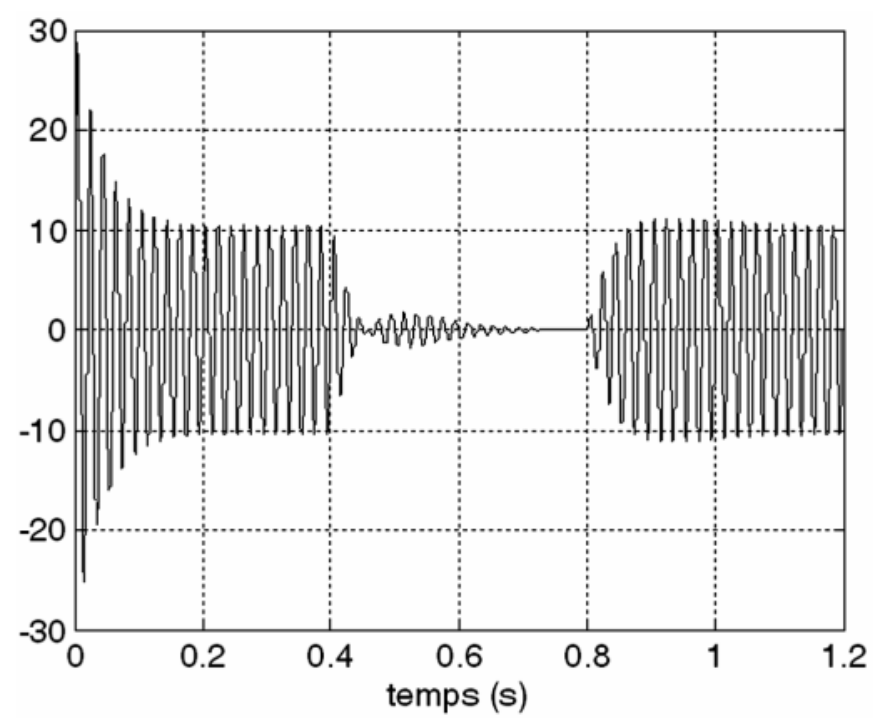

Fig. 7: Input Current $x_{1}$

\section{CONCLUSION}

In this paper we have considered the problem of controlling a full-bridge rectifier of the boost type. The converter dynamics have been described by the average 2 th order nonlinear state-space model (1). Based on such model, a cascade nonlinear controller has been designed using the sliding mode technique. It has been formally established, using Lyapunov stability and averaging theory, that the obtained controller meets its objectives.

\section{REFERENCES}

[1] B. Lin and H. Lu. "Single-phase Power-factor-correction ac/dc Converters with three PWM Control Schemes". IEEE Trans. on Aerospace and Electronic Systems, 36, 189-200, January 2000.

[2] Redl R., (1994). "Power-factor Correction in Single-phase Switching-mode Power Supplies". Int. J. Electron., 77, pp. 555-582.

[3] Tse C.K. and M.H.L. Chow (2000). "Theoretical Study of Switching Converters with Power Factor Correction and Output Regulation". IEEE Trans. Circuits Syst. I, 47, pp. 1047-55.

[4] Escobar G., D. Chevreau, R. Ortega and E. Mendes, (2001). "An Adaptive Passivity-based Controller for a Unity Power Factor Rectifier". IEEE Trans. Contr. Syst. Technol., 9, pp. 637-644.

[5] Spiazzi G., P. Mattavelli and L. Rossetto, (1995). “Power Factor Preregulators with Improved Dynamic Response". Proceedings of IEEE Power Electron. Spec. Conf. (PESC), 1, pp. 150-156.

[6] Giri F., A. Abouloifa and I. Lachkar, (2005). “Nonlinear Control of Boost AC/DC Converters". IFAC World Congress, Prague, Czech Republic, July 4-8.

[7] Khalil H., (2003). "Nonlinear Systems", Prentice-Hall, NJ, USA.

[8] Zhi-Fen Z., D. Tong-Ren, H. Wen-Zao and D. Zhen-Xi, (1992). "Qualitative Theory of Differential Equations". American Mathematical Society, Rhode Island, USA.

[9] N. Mohan, T.M. Undeland, and W.P. Robbins. "Power Electronics. Converters,Applications, and Design". John Wiley and Sons, 1995.

[10] Mechi A. and S. Funabiki, (1993). "Step-up/Down Voltage PWM AC to DC Convector with one Switching Device". IEE proceedings-B, 140, No. 1, pp. 35-43.

[11] Karagiannis D.E., Mendes A., Astolfi, and R. Ortega, (2003). An Experimental Comparison of Several PWM Controllers for a Single-phase AC-DC Converter". IEEE Trans. Contr. Syst. Technol., 11, pp. 940-947.

[12] Abouloifa A., F. Giri, and I. Lachkar, (2003). “Nonlinear Control of a PWM Rectifier Output Voltage Regulation and Power Factor Correction". Proceedings of the 5th Int. Symp. on Advanced Electromechanical Motion Systems (ELECTROMOTION), pp. 2-7.

[13] H. Sira-Ramirez. "Sliding Motions in Bilinear Switched Networks". IEEE Trans. on Circuit and Systems, (34), 919-933, 1987.

[14] V.I. Utkin. "Sliding Modes and their Applications in Variable Structure Systems". Mir, Moscow, 1978. 